\title{
Recent advances in the Lefschetz fixed point theory for multivalued mappings
}

\author{
Lech Górniewicz ${ }^{\mathrm{a}}$
}

Dedicated to my teacher Professor Kazimierz Gêba

${ }^{a}$ Faculty of Mathematics and Computer Science, Nicolaus Copernicus University in Toruń, Chopina 12/18, 87-100 Toruń, Poland.

\begin{abstract}
In 1923 S. Lefschetz proved the famous fixed point theorem known as the Lefschetz fixed point theorem (comp. [5], [9], 20], 21]. The multivalued case was considered for the first time in 1946 by S. Eilenberg and D. Montgomery ([10]). They proved the Lefschetz fixed point theorem for acyclic mappings of compact ANR-spaces (absolute neighbourhood retracts (see [4] or [13]) using Vietoris mapping theorem (see [4], [13], [16]) as a main tool. In 1970 Eilenberg, Montgomery's result was generalized for acyclic mappings of complete ANR-s (see [17]). Next, a class of admissible multivalued mappings was introduced ([13] or [16]). Note that the class of admissible mappings is quite large and contains as a special case not only acyclic mappings but also finite compositions of acyclic mappings. For this class of multivalued mappings several versions of the Lefschetz fixed point theorem was proved (comp. [11], [13]-[15], [18], [19], [27]). In 1982 G. Skordev and W. Siegberg ([26]) introduced the class of multivalued mappings so-called now $(1-n)$-acyclic mappings. Note that the class $(1-n)$-acyclic mappings contains as a special case $n$-valued mappings considered in [6], [12], [28]. We recommend [8] for the most important results connected with $(1-n)$-acyclic mappings. Finally, the Lefschetz fixed point theorem was considered for spheric mappings (comp. [3], [2], [7], [23]) and for random multivalued mappings (comp. [1], 2], [13]). Let us remark that the main classes of spaces for which the Lefschetz fixed point theorem was formulated are the class of ANR-spaces ([4]) and MANR-spaces (multi absolute neighbourhood retracts (see [27]). The aim of this paper is to recall the most important results concerning the Lefschetz fixed point theorem for multivalued mappings and to prove new versions of this theorem, mainly for AANR-spaces (approximative absolute neighborhood retracts (see [4] or [13]) and for MANR-s. We believe that this article will be useful for analysts applying topological fixed point theory for multivalued mappings in nonlinear analysis, especially in differential inclusions.
\end{abstract}

Email address: gorn@mat.umk.pl (Lech Górniewicz) 
Keywords: multivalued mappings, fixed point problem, absolute retracts, admissible mappigns, $(1-n)$-acyclic mappings, spheric and random mappings, Lefschetz number.

2020 MSC: Primary: 54H25, 55N20, 47H1154C55, 54C60; Secondary: 47H04, 47H10, 47H40, 55M15, $28 \mathrm{~B} 20$.

\section{Auxiliary notions}

In this paper all topological spaces are assumed to be metric. We shall consider the Čech homology functor with compact carriers and coefficients in the field of rational numers $Q$ (for details see [13] or [16]). For a space $X$ by $H(X)=\left\{H_{n}(X) ; n \geq 0\right\}$ we shall denote the Čech homology with compact carriers and coefficients in $Q$ of the space $X$. Then $H_{n}(X)$ is a linear vector space over $Q$.

For any continuous map $f: X \rightarrow Y$ by $f_{*}=\left\{f_{* n} ; n \geq 0\right\}: H(X)=\left\{H_{n}(X) ; n \geq 0\right\} \rightarrow H(Y)=$ $\left\{H_{n}(X) ; n \geq 0\right\}$ we denote the induced linear map, i.e. $f_{* n}: H_{n}(X) \rightarrow H_{n}(Y)$ is a linear map, $n \geq 0$.

Definition 1.1. A space $X$ is called of finite type provided:

(a) the dimension $\operatorname{dim} H_{n}(X)<+\infty$ for every $n \geq 0$,

(b) $H_{n}(X)=0$ for almost all $n$.

Definition 1.2. A space $X$ is called acyclic provided $H_{0}(X)=Q$ and $H_{n}(X)=0$, for every $n \geq 1$.

Evidently, any contractible space is acyclic; an acyclic space is of finite type.

Definition 1.3. A continuous map $p: Y \rightarrow X$ is called Vietoris map provvvided:

(a) $p$ is onto,

(b) for every compact set $K \subset X$ the counter image $p^{-1}(K)$ is compact,

(c) $p^{-1}(x)$ is acyclic for every $x \in X$.

Theorem 1.4 (Vietoris Mapping Theorem, [4], [13], [16]). If $p: Y \rightarrow X$ is a Vietoris map, then $p_{* n}: H_{n}(Y) \rightarrow$ $H_{n}(X)$ is a linear isomorphism for every $n \geq 0$.

In what follows if $p: Y \rightarrow X$ is a Vietoris map then shall use the following symbol: $p: Y \Longrightarrow X$. Let us consider the following diagram:

$$
X \stackrel{p}{\longleftarrow} Y \stackrel{q}{\longrightarrow} X
$$

Then, for every $n \geq 0$, we have the following diagram of linear mappings:

$$
H_{n}(X) \stackrel{p_{* n}}{\sim} H_{n}(Y) \stackrel{q_{* n}}{\longrightarrow} H_{n}(X)
$$

in which $p_{* n}$ is an isomorphism. So we can define the following linear map:

$$
q_{* n} \circ p_{* n}^{-1}: H_{n}(X) \rightarrow H_{n}(X)
$$

which is called by us the induced by the pair $(p, q)$ linear map, for every $n \geq 0$.

Now we are going to define the Lefschetz number. First let assume that $E$ is a linear vector space over $Q$ and $l: E \rightarrow E$ a linear endomorphism.

(1) Assume further that $\operatorname{dim} E<+\infty$, then by $\operatorname{tr}(l)$ we shall denote the trace of $l$.

(2) Assume that $\operatorname{dim} E=+\infty$. Denote by $l^{(n)}: E \rightarrow E$ the $n$-th iterate of $l$ and observe that

$$
\operatorname{Ker}(l) \subset \operatorname{Ker}\left(l^{(2)}\right) \subset \ldots \subset \operatorname{Ker}\left(l^{(n)}\right) \subset \ldots
$$


We put

$$
N(l)=\bigcup_{n=1}^{+\infty} \operatorname{Ker}\left(l^{(n)}\right) \quad \text { and } \quad \widetilde{E}=\left.E\right|_{N(l)} .
$$

Since $N(l)$ is an invariant subspace of $E$ we can consider the induced endomorphism $\widetilde{l}: \widetilde{E} \rightarrow \widetilde{E}$. We shall say that $l$ is a Leray endomorphism provided $\operatorname{dim} E<+\infty$. Consequently we are able to define the generalized trace $\operatorname{Tr}(l)$ of $l$ by the formula:

$$
\operatorname{Tr}(l)=\operatorname{tr}(\widetilde{l}) .
$$

Consider a continuous mapping $f: X \rightarrow X$. We shall say that the Lefschetz number $\lambda(f)$ of $f$ is well defined provided $f_{* n}$ is a Leray endomorphism for every $n \geq 0$ and $\operatorname{Tr}\left(f_{* n}\right)=0$ for almost all $n$. Then we let:

$$
\lambda(f)=\sum_{n=1}^{+\infty}(-1)^{n} \operatorname{Tr}\left(f_{* n}\right) .
$$

Evidently, if $X$ is a space of finite type, then

$$
\lambda(f)=\sum_{n=0}^{+\infty}(-1)^{n} \operatorname{tr}\left(f_{* n}\right)
$$

is well defined. Observe that for given pair $(p, q)$ the above consideration is exactly the same and we have

$$
\lambda((p, q))=\sum_{n=0}^{+\infty}(-1)^{n} \operatorname{Tr}\left(q_{* n} \circ p_{* n}^{-1}\right)
$$

provided it is well defined.

The following proposition is very usefull in what follows:

Proposition 1.5. Assume that the following diagram of linear mappings

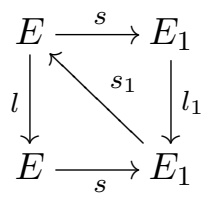

is commutative. Then $l$ is a Leray endomorphism if and only if $l_{1}$ is a Leray endomorphism and in that case

$$
\operatorname{Tr}(l)=\operatorname{Tr}\left(l_{1}\right) .
$$

For the proof of Proposition 1.5 see [13], [16], [20]. Note that Proposition 1.5 can be formulated in the terms of continuous mappings and the Lefschetz number (see again [13], [16], [20]).

Finally assume that $l: E \rightarrow E$ is a weakly nilpotent map, i.e. for all $x \in E$ there exists $n_{x}>0$ suth that $l^{n_{x}}(x)=0$. We have (see [13]):

Proposition 1.6. If $l: E \rightarrow E$ is a weakly nilpotent endomorphism, then $\operatorname{Tr}(l)=0$.

\section{Multivalued mappings}

Let $\mathrm{X}$ and $Y$ be two metric spaces. A multivalued map defined on $X$ with the values in $Y$ we shall denote by the symbol $\varphi: X \multimap Y$. In what follows we shall denote multivalued mappings by greek letters. We shall assume also that, for every $x \in X$ the value $\varphi(x)$ of $x$ under $\varphi$ is a non empty subset of $Y$.

Let $\varphi: X \multimap Y$ be a multivalued mapping. We associate with $\varphi$ the graph $\Gamma_{\varphi}$ by putting:

$$
\Gamma_{\varphi}=\{(x, y) \in X \times Y ; y \in \varphi(x)\} .
$$


Then we have the following diagram:

$$
X \stackrel{p_{\varphi}}{\longleftarrow} \Gamma_{\varphi} \stackrel{q_{\varphi}}{\longrightarrow} Y
$$

in which $p_{\varphi}(x, y)=x$ and $q_{\varphi}(x, y)=y$ for every $(x, y) \in \Gamma_{\varphi}$.

A multivalued map $\varphi: X \multimap Y$ is called upper semicontinuous (u.s.c.) provied for every open set $U \subset Y$ the set

$$
\varphi^{-1}(U)=\{x \in X ; \varphi(x) \subset U\}
$$

is open in $X ; \varphi$ is called lower semicontinuous (l.s.c.) provided for every open set $U \subset Y$ the following set:

$$
\varphi_{+}^{-1}(U)=\{x \in X ; \varphi(x) \cap U \neq \emptyset\}
$$

is open in $X$.

Finally, $\varphi$ is called continuous if and only if $\varphi$ is both u.s.c. and l.s.c. A mapping $\varphi: X \multimap Y$ is called compact provided $\varphi(X)=\bigcup_{x \in X} \varphi(x)$ is relatively compact subset of $Y$.

Remark 2.1. In what follows we shall consider only u.s.c. mappings with compact values.

Definition 2.2. An u.s.c. mapping $\varphi: X \multimap Y$ is called acyclic provided $\varphi(x)$ is an acyclic set for every $x \in X$.

The following two remarks are selfevident.

Remark 2.3. If $\varphi: X \multimap Y$ is an acyclic map then $p_{\varphi}$ is a Vietoris map.

Remark 2.4. Moreover, we have $\varphi(x)=q_{\varphi}\left(p_{\varphi}^{-1}(x)\right)$, for every $x \in X$; the pair $\left(p_{\varphi}, q_{\varphi}\right)$ will be called a selective pair of $\varphi$ (written $\left(p_{\varphi}, q_{\varphi}\right) \subset \varphi$.

For given two maps: $X \stackrel{\varphi}{\circ} Y \stackrel{\psi}{\circ} Z$ we define the composition $\psi \circ \varphi$ of $\varphi$ and $\psi$ by the formula:

$$
(\psi \circ \varphi)(x)=\bigcup\{\psi(y) ; y \in \varphi(x)\}
$$

for every $x \in X$. Observe that the composition of two acyclic maps is not an acyclic map in general.

Now we are going to define the notion of admissible multivalued maps (see [13], [16]).

Definition 2.5 ([13], [16]). A multivalued map $\varphi: X \multimap Y$ is called admissible provided there exists a diagram

$$
X \stackrel{p}{\longleftarrow} Z \stackrel{q}{\longleftrightarrow} Y
$$

in which $p$ is a Vietoris map and $q$ is continuous such that

$$
\varphi(x)=q\left(p^{-1}(x)\right) \quad \text { for every } x \in X .
$$

Evidently, (comp. Remark 2.4 any acyclic map is admissible. Moreover, the following property holds true.

Property 2.6. Compositions of two admissible mappings is admissible too.

Note that the class of acyclic maps was introduced by S. Eilenberg and D. Montgomery in 1946 (see [10]); the class of admissible mappings was introduced in 1976 (see [16]). 


\section{Appropriate spaces}

In this section we recall types of metric spaces essential from the point of view of Lefschetz fixed point theorem. Let $A$ be a closed subset of the space $X$. We shall say that $A$ is a retract of $X$ provided there exists a continuous map $r: X \rightarrow A$ (called retraction map) such that $r(x)=x$, for every $x \in X$; the map $r_{\varepsilon}: X \rightarrow A$ is called $\varepsilon$-retraction provided $d\left(r_{\varepsilon}(x), x\right)<\varepsilon$ for every $x \in A$, where $d$ denotes the metric in $X$.

Definition 3.1. We shall say that a space $X$ is an absolute retract $(X \in \mathrm{AR})$ provided, for every space $Y$ and for every embedding $h: X \rightarrow Y$ as aclosed subset, the set $h(X)$ is a retract of $Y$; if there exists an open $U \subset Y$ such that $A \subset U$ is a retract of $U$, then $X$ is called an absolute neighbourhood retract ( $X \in$ ANR).

Note that any metric space $X$ can be embedded as a closed subset into a normed space. Moreover, let us add that any absolute retract is a contractible space (see [4], [13, [20]).

Definition 3.2. A map $r: X \rightarrow Y$ is called multi-retraction provided there exists an admissible map $\varphi: Y \multimap$ $X$ such that $r(\varphi(y))=y$, for every $y \in Y$.

Definition 3.3 ([27]). A space $X$ is called an absolute multiretract ( $X \in \mathrm{AMR}$ ) provided there exists a normed space $E$ and a multi-retraction $r: E \rightarrow X ; X$ is called an absolute neighbourhood multi-retrac $(X \in \mathrm{ANMR})$ provided there exists an open subset of some normed space $E$ and a multi-retraction $r: U \rightarrow X$.

The following diagram illustrates the relationship between the introduced spaces:

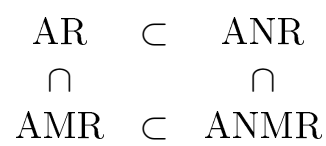

Note that all of the above inclusions are proper. Moreover, let us add that every $X \in \mathrm{AMR}$ is an acyclic space.

Finally, we shall recall notions of approximative retracts.

Definition $3.4([4],[13],[16])$. A space $X$ is called an absolute approximative retract provided for any embedding $h: X \rightarrow Y$ of $X$ into a space $Y$ and, for every $\varepsilon>0$, there exists an $\varepsilon$-retraction $r_{\varepsilon}: Y \rightarrow h(X)$ (written $X \in \mathrm{AAR}$ ); if there exists an open subset $U$ of $X$ such that, for every $\varepsilon>0$ tehre is an $\varepsilon$-retraction $r_{\varepsilon}: U \rightarrow h(X)$, then $X$ is called an absolute approximative neighbourhood retract ( $X \in \mathrm{AANR}$ ).

It $\mathrm{s}$ easy to see that

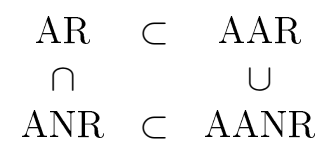

and all inclusions are proper (comp. [13]).

\section{The Lefschetz fixed point theorem for admissible mappings}

First we remind definitions of some types of admissible mappings. We let

$$
K(X)=\{\varphi: X \multimap X ; \varphi \text { is admissible and compact }\} .
$$

Definition 4.1. Let $\varphi: X \multimap X$ be admissible mapping.

(a) $\varphi$ is called compact absorbing contraction $(\varphi \in \operatorname{CAC}(X))$, if there exists an open subset $U$ of $X$ such that $\overline{\varphi(X)}$ is a compact subset of $U$ and, for every $x \in X$ there exists a natural number $n_{X}>0$ such that $\varphi^{n_{X}}(x) \subset U$; 
(b) $\varphi$ os called eventually compact if $\varphi^{n} \in \mathrm{K}(X)$, for some $n>0$;

(c) $\varphi$ is called asymptotically compact provided, for every $x \in X$, the orbit $O(x)=\bigcup_{n=1}^{\infty} \varphi^{n}(x)$ is relatively compact and the core $\mathrm{C}(\varphi)=\bigcap_{n \geq 1} \varphi^{n}(X)$ is nonempty relatively compact $(\varphi \in \operatorname{ASC}(X))$;

(d) $\varphi$ is called compact attraction if there exists a compact subset $K \subset X$ such that, for every open neighbourhood $V$ of $K$ in $X$ such that, for every $x \in X$, there exists $n_{X} \geq 0$ for which $\varphi^{n_{X}}(x) \subset V$ $(\varphi \in \mathrm{CA}(X))$.

Note that the notion of CAC-mappings was introduced in [11]. We have:

$$
\mathrm{K}(X) \subset \mathrm{EC}(X) \subset \operatorname{ASC}(X) \subset \mathrm{CA}(X) \subset \mathrm{CAC}(X) .
$$

Let us add that all the above inclusions are proper (see [13], [19], [25]).

Let $\varphi: X \multimap X$ be an addmissible map and $(p, q) \subset \varphi$ be a selective pair of $\varphi$. Then we have the induced linear maps:

$$
q_{* n} \circ p_{* n}^{-1}: H_{n}(X) \rightarrow H_{n}(X),
$$

for every $n$. So we shall say that $(p, q)$ is a Lefschetz pair provided $q_{* n} \circ p_{* n}^{-1}$ is a Leray endomorphism for every $n \geq 0$ and $\operatorname{Tr}\left(q_{* n} \circ p_{* n}^{-1}\right)=0$ for almost all $n \geq 0$. Consequently in this case we have well defined the Lefschetz number $\lambda((p, q))$ od the pair $(p, q)$ by the formula 1.4 .

We shall say that $\varphi$ is a Lefschetz map provided every selected pair $(p, q) \subset \varphi$ of $\varphi$ is a Lefschetz pair. In this case we define the Lefschetz set $\Lambda(\varphi)$ of $\varphi$ by putting:

$$
\Lambda(\varphi)=\{\lambda(p, q) ;(p, q) \subset \varphi\} .
$$

Note that, if $X$ is of finite type, then for any admissible map $\varphi$ the Lefschetz set $\Lambda(\varphi)$ of $\varphi$ is well defined. Observe that, if $\varphi$ is acyclic map and $X$ is of finite type, then the Lefschetz set $\Lambda(\varphi)$ of $\varphi$ is a singleton, i.e. $\Lambda(\varphi)=\left\{\lambda\left(p_{\varphi}, q_{\varphi}\right)\right\}$

Finally, it is easy to see that, if $X$ is an acyclic space, then for every admissible map $\varphi: X \multimap X$ is a Lefschetz map and $\Lambda(\varphi)=\{1\}$, i.e. for every $(p, q) \subset \varphi$, we have $\lambda(p, q)=1$. We recommend for the details [13], [16], [19].

The following theorem is provided in [27].

Theorem 4.2. If $X \in \mathrm{MANR}$ and $\varphi \in \mathrm{K}(X)$, then $\varphi$ is a Lefschetz map and $\Lambda(\varphi) \neq\{0\}$ implies that there exists a fixed point of $\varphi$, i.e. there exists $x \in X$ such that $x \in \varphi(x)$.

Note that, for $X \in \mathrm{ANR}$, Theorem 4.2 was proved in [11] and for $X$ to be compact ANR-space and $\varphi: X \multimap X$ to be acyclic map it was proved in [10].

Now we shall present essential generalization of Theorem 4.2

Theorem 4.3. If $X \in \mathrm{MANR}$ and $\varphi \in \mathrm{CAC}(X)$, then $\varphi$ is Lefschetz map and $\Lambda(\varphi) \neq\{0\}$ implies that $\varphi$ has a fixed point.

Proof. Since $\varphi \in$ CAC there exists an open subset $U \subset X$ such that $\overline{\varphi(X)}$ is a compact subset of $U$. Consequently the map $\varphi^{\prime}: U \multimap U, \varphi^{\prime}(x)=\varphi(x)$ for every $x \in U$ is an admissible compact map. Moreover, $\left(p^{\prime}, q^{\prime}\right) \subset \varphi^{\prime}$, where $p^{\prime}, q^{\prime}: p^{-1}(U) \rightarrow U$ are defined as follows: $p^{\prime}(u)=p(u), q^{\prime}(u)=q(u)$ for every $u \in p^{-1}(U)$ and the arbitrary selected pair $(p, q) \subset \varphi$.

To show that $\varphi$ is a Lefschetz map it is enough to prove that $\Lambda(p, q)=\Lambda\left(p^{\prime}, q^{\prime}\right)$. In view of Theorem 4.2 we obtain that the Lefschetz number $\Lambda\left(\left(p^{\prime}, q^{\prime}\right)\right)$ is well defined. In order to show that $\Lambda((p, q))=\Lambda\left(\left(p^{\prime}, q^{\prime}\right)\right)$ we consider pairs $(X, U)$ and $(\bar{p}, \bar{q})$, where $\bar{p}, \bar{q}:\left(p^{-1}(X), p^{-1}(U)\right) \rightarrow(X, U)$ are defined by

$$
\bar{p}(u)=p(u), \quad \bar{q}(u)=q(u), \quad \text { for every } u \in p^{-1}(X) .
$$


Now we are able to define a linear map $l_{* n}: H_{n}(X, U) \rightarrow H_{n}(X, U)$ defined by the formula

$$
l_{* n}=\bar{q}_{* n} \circ \bar{p}_{* n}^{-1} \quad \text { for every } n \geq 0 .
$$

Since $\varphi \in \operatorname{CAC}(X)$ we deduce that $l_{* n}$ is weakly nilpotent, for every $n \geq 0$. Hence $\operatorname{Tr}\left(l_{* n}\right)=0$ for every $n \geq 0$. Now using homology exact sequence (see [13]) we obtain that

$$
\operatorname{Tr}\left(q_{* n} \circ p_{* n}^{-1}\right)=\operatorname{Tr}\left(q_{* n}^{\prime} \circ\left(p_{* n}^{\prime}\right)^{-1}\right) \quad \text { for every } n \geq 0
$$

and hence $\Lambda((p, q))=\Lambda\left(\left(p^{\prime}, q^{\prime}\right)\right)$.

Finally, if we assume that $\Lambda((p, q)) \neq\{0\}$, then $\Lambda\left(\left(p^{\prime}, q^{\prime}\right)\right) \neq 0$ and from Theorem 4.2 we obtain that $\varphi^{\prime}$ has a fixed point. But every fixed point of $\varphi^{\prime}$ is also a fixed point of $\varphi$ and our theorem is proved.

Note that theorem was formulated in [18] but witout the proof. For $X \in$ ANR Theorem 4.2 was proved in [11] (see also [13]).

Corollary 4.4. Assume that $X$ satisfies one of the following three conditions:

(a) $X$ is an acyclic MANR-space,

(b) $X \in \mathrm{MAR}$,

(c) $\in$ AR.

If $\varphi \in \mathrm{CAC}(X)$, then $\varphi$ has a fixed point.

In the end of this section we shall deal with approximative retracts. First we remind the following fact:

Proposition 4.5 ([16]). If $X$ is a compact AANR-space, then it is of finite type.

The key in the proof the Lefschetz fixed point theorem is the following:

Proposition 4.6 ([16]). Assume that $y$ is a compact space of finite type. Then there exists $\varepsilon>0$ such that for arbitrary space $Y$ and two conditions maps $f, g: Y \rightarrow Y$ if $d(f(y), g(y))<\varepsilon$, for every $y \in Y$, then $f_{* n}=g_{* n}$, for every $n \geq 0$.

Theorem 4.7 ([13], [16]). Let $X$ be a compact AANR-space and $\varphi: X \multimap X$ an admissible map. Then $\Lambda(\varphi) \neq 0$ implies that $\varphi$ has a fixed point.

Corollary 4.8. If $X$ is an acyclic AANR or $X \in \mathrm{AAR}$, then for every admissible map $\varphi: X \multimap X$ there exists a fixed point.

Open Problem 4.9. Is it possible to prove Theorem 4.7 for arbitrary $X \in$ AANR and $\varphi \in K(X)$ or $\varphi \in \mathrm{CAC}(\mathrm{X}) ?$

There exists a large literature concerning the Lefschetz fixed point theorem for acyclic and admissible mappings (comp. [2], [5], [9], [10], [13]-[19], [21], 25], [27]) and references there in.

\section{Random admissible operators}

A systematic study of random operation was iniciated in the 1950 by Czech mathematicians. We are interested in the fixed point theory for multivalued random operators. For more details and references sii [1] or [13] and references there in.

By a measurable space we shall mean the pair $(\Omega, \Sigma)$, where the set $\Omega$ is equipped in a $\sigma$-algebra $\Sigma$ of subsets. We shall use $B(X)$ to denote the Borel $\sigma$-algebra on $X$. The symbol $\Sigma \otimes B(X)$ denotes the smallest $\sigma$-algebra on $\Omega \times X$ which contains all the sets $A \times B$, where $A \in \Sigma$ and $B \in B(X)$. 
Definition 5.1. Let $(\Omega, \Sigma)$ be a measurable space and $Y$ a separable space. A map $\varphi: \Omega \multimap X$ with closed values is called measurable if

$$
\varphi^{-1}(B)=\{\omega \in \Omega ; \varphi(\Omega) \subset B\} \in \Sigma \text { for each open } B \subset Y .
$$

Definition 5.2. A multivalued map $\varphi: \Omega \times X \multimap X$ with closed values is called random map provided:

(a) $\varphi$ is product measurable (measurable in the whole),

(b) the map $\varphi(\omega, \cdot): X \multimap X$ is u.s.c. for every $\omega \in \Omega$,

(c) if the map $\varphi(\omega, \cdot): X \multimap X$ is admissible, then $\varphi$ is called random admissible map.

Definition 5.3. Let $\varphi: \Omega \times X \multimap X$ be a random map. A measurable map (singlevalued) $\xi: \Omega \rightarrow X$ is called a random fixed point of $\varphi$ provided:

$$
\xi(\omega) \in \varphi(\omega, \xi(\omega)), \quad \text { for every } \omega \in \Omega .
$$

The following proposition is crucial in what follows.

Proposition 5.4 ([1], [13]). Let $X$ be a separable space and $\varphi: \Omega \times X \multimap X$ a random operator such that the map $\varphi(\omega, \cdot): X \multimap X$ has a fixed point for every $\omega \in \Omega$, then $\varphi$ has a random fixed point.

Now we are able to prove the main result of this section, i.e. the Lefschetz-type fixed point theorem for random maps.

Theorem 5.5. Assume that $X$ is a separable multi absolute neighbourhood retract $(X \in \mathrm{MANR})$. Assume further that $\varphi: \Omega \times X \multimap X$ is a random admissible map such that $\varphi(\omega, \cdot) \in \operatorname{CAC}(X)$ for every $\omega \in \Omega$. If $\lambda(\varphi(\omega, \cdot) \neq\{0\}$, for every $\omega \in \Omega$, then $\varphi$ has a random fixed point.

Proof. In view of Theorem $4.3 \varphi(\omega, \cdot)$ is a Lefschetz map for every $\omega \in \Omega$. Consequently our assumption that $\Lambda(\varphi(\omega, \cdot)) \neq\{0\}$ implies that $\varphi(\omega, \cdot)$ has a fixed point. Hence our theorem follows from Proposition 5.4 and the proof is complete.

Corollary 5.6. Assume that $X$ is a separable and one of the following conditions is satisfied:

(a) $X$ is acyclic multi absolute neighbourhood retract,

(b) $X \in \mathrm{AR}$,

(c) $X \in \mathrm{ANR}$ and it is acyclic,

then an random admissible map $\varphi: \Omega \times X \multimap X$ such that $\varphi(\omega, \cdot) \in \mathrm{CAC}(X)$, for every $\omega \in$ Omega has a random fixed point.

The formulation of the respective Lefschetz-type theorem for $X$ to be compact AANR-space is left to the reader.

\section{Spheric maps}

In 1947 B. O'Neill [24] constructed an example of a fixed point free map $\varphi: K^{2} \multimap K^{2}$ which is continuous with values homotopically equivalent to $S^{1}$ (even homeomorfic to $S^{n}$ ), where $K^{2}$ denotes the unit ball in euclidean space $R^{2}$ and $S^{1}$ denotes the unit sphere. The above example was inspiration to introduce the notion of spheric mappings. For spheric mappings see [3], [7, [13], [23]. In this section all considered topological spaces are subsets of the euclidean space $R^{n}, n \geq 1$.

Let $X$ be a compact subsets of $R^{n}, n \geq 2$. Then $R^{n} \backslash X$ consists of two components, namely: 
(1) an unbounded component $D X$ of $R^{n} \backslash X$, i.e. $x \in D X$ if and only if for every $r>0$ there exists a continuous function $f:[0,1] \rightarrow R^{n} \backslash X$ such that $f(0)=x$ and $\|f(1)\|>r$, since $X$ is compact $D X$ is the unique unbounded component of $R^{n} \backslash X$.

(2) a union of all bounded component $B X$ of $\left(R^{n} \backslash D X\right) \backslash X$. Moreover, we let

$$
\widetilde{X}=B X \cup X=R^{n} \backslash D X .
$$

Observe that if $A$ is a compact subset of $X$, then $\widetilde{A} \subset \widetilde{X}$; if $X$ is an acyclic set then $X=\widetilde{X}$.

Definition 6.1. Let $X$ be a compact subset of $R^{n}$ and let $\varphi: X \multimap X$ be an u.s.c. map with connected values. We say that $\varphi$ is a spheric map provided:

(a) $\{x \in X ; x \in B \varphi(x)\}$ is an open subset of $X$.

(b) the map $\widetilde{\varphi}: X \multimap X, \widetilde{\varphi}(x)=\varphi(x) \cup B \varphi(x)$ is well defined acyclic map.

Of course any acyclic map is spheric. Evidently, if $X$ is an acycylic set or $R^{n} \backslash X$ is connected, then $\widetilde{\varphi}$ is well defined.

Let $\varphi: X \multimap X$ be a spheric map. Then $\widetilde{\varphi}: X \multimap X$ is acyclic map and consequently the linear map $\widetilde{\varphi}_{* n}: H_{n}(X) \rightarrow H_{n}(X)$, given by the formula

$$
\widetilde{\varphi}_{* n}\left(q_{\widetilde{\varphi}}\right)_{* n}\left(p_{\widetilde{\varphi}}\right)_{* n}^{-1}, \quad n \geq 0 .
$$

is well defined for every $q \geq 0$. We define the Lefschetz number $\lambda(\varphi)$ of $\varphi$ by putting: $\lambda(\varphi)=\lambda(\widetilde{\varphi})$, provided $\lambda(\widetilde{\varphi})$ is well defined. The following proposition is crucial.

Proposition 6.2. If $\varphi: X \multimap X$ is a spheric map and $\widetilde{\varphi}: X \multimap X$ has a fixed point, then $\varphi$ has a fixed point.

Proof. There are possibilities:

(i) the boundary $\partial X$ of $X$ in $R^{n}$ is equal to $X$,

(ii) $\partial X \neq X$.

In the case (i) we have $\varphi=\widetilde{\varphi}$ so our claim is evident.

For the proof of (ii) assume to the contrary that $\varphi$ has no fixed points. Then the set Fix $(\widetilde{\varphi})=\{x \in$ $X ; x \in \widetilde{\varphi}(x)\}$ is closed subset of $X$ and in view of Definition 6.1 we deduce that Fix $(\widetilde{\varphi})$ is also an open subset. To obtain a contradiction it is sufficent to observe that $X \backslash\{x \in X ; x \in B \varphi(x)\} \neq \emptyset$. Indeed, we have that $\partial X \neq \emptyset$ (since $X$ is compact) if $x \in \partial X$ then $x \notin B \varphi(x)$ and hence $X \backslash\{x \in X ; x \in B \varphi(x)\} \neq \emptyset$ and the proof is completed.

From Proposition 6.2 and Theorems 4.2 , 4.7 we obtain

Theorem 6.3. If $X$ is a compact subset of $R^{n}$ and $X \in \mathrm{MANR}$ or $X \in \mathrm{AANR}$ and $\varphi: X \multimap X$ is a spheric map, then $\lambda(\varphi) \neq 0$ implies that $\varphi$ has a fixed point.

Corollary 6.4. In particular, if $X \in \mathrm{MAR}$ or $X \in \mathrm{AR}$ or $X \in \mathrm{AAR}$, then any spheric map $\varphi: X \multimap X$ has a fixed point.

Remark 6.5. Evidently the respective results for random spheric maps are also possible (comp. Section 5). 


\section{7. $(1-n)$-acyclic mappings}

Observe that both admissible and spheric mappings have connected values. In this section we shall present a class of mappings with nonconnected values.

Definition 7.1. A continuous multivalued map $\varphi: X \multimap X$ is called $(n-1)$-acyclic $\left(\varphi \in A_{(1, n)}(X)\right)$ provided for every $x \in X$ the value $\varphi(x)$ consists from one or $n$ compact acycylic components ( $n$ is fixed).

In 1957 B. O'Neill claimed the existence of a finitely-valued continuous fixed point free maps $\varphi: K^{2} \multimap K^{2}$ but gave not example. In 1982 in [22] there is presented fixed point free map $\varphi: K^{2} \rightarrow K^{2}$ with 1 or 2 or 3 values. Of course the above $\varphi \notin A_{(1-3)}$.

Let us remark that the class of $(1-n)$-acyclic mappings is still quite large, namely:

(i) if $\varphi$ is continuous and acyclic, then $\varphi \in A_{(1-1)}(X)$,

(ii) if $\varphi$ is $n$-valued map, i.e. $\varphi(x)$ consists from exactly $n$ points, then $\varphi \in A_{(1-n)}(X)$ (see [6], [12]),

(iii) if $\varphi$ is $n$-valued split map in the sense of [12], then it is $(1-n)$-acyclic.

It is possible to define the Lefschetz number of a map $\varphi \in A_{(1-n)}(X)$ by associating to this map a chain map (chain approximation). For details see [8], [13], [26].

Consequently it is possible to prove the Lefschetz fixed point theorem. Namely, we have:

Theorem $7.2\left(\left[8\right.\right.$, 13]). Assume that $X \in \mathrm{ANR}$ and $\varphi \in A_{(1-n)}(X) \cap \mathrm{CAC}(X)$. Then the Lefschetz number $\lambda(\varphi) \neq\{0\}$ implies that $\varphi$ has a fixed point.

Corollary 7.3. If $X \in \mathrm{AR}$ and $\varphi \in A_{(1-n)}(X) \cap \mathrm{CAC}(X)$, then $\varphi$ has a fixed point.

\section{Open Problems 7.4.}

(a) Is it possible to prove Theorem 7.2 for $X \in$ MANR?

(b) Is it possible to prove Theorem 7.2 for $X \in \mathrm{AANR}$ ?

Finally, let us add that Theorem 7.2 can be randomized.

\section{References}

[1] J. Andres, L. Górniewicz, On the Lefschetz fixed point theorem for radom multivalued mappings, Lib. Math. (N.S.) (2013), 69-78.

[2] J. Andres, L. Górniewicz, Recent results on the topological fixed point theory of multivalued mappings: a survey, Fixed Point Theory Appl. 184 (2015), 1-34.

[3] J. Andres, L. Górniewicz, Lefschetz-type fixed point theorem for spheric mappings, Fixed Point Theory 19 (2018), no. 2, $453-461$.

[4] K. Borsuk, Theory of Retracts, PWN, Warszawa 1967.

[5] R. Brown, The Lefschetz Fixed Point Theorem, London 1971.

[6] R. Brown, The Lefschetz number of an $n$-valued multimap, Fixed Point Theory Appl. 2 (2007), 53-60.

[7] A. Dawidowicz, Spherical maps, Fund. Math. 127 (1987), 187-196.

[8] Z. Dzedzej, Fixed Point Index for a class of nonacyclic multivalued maps, Dissertationes Math. 25 (1985).

[9] O. Ege, I. Karaca, Lefschetz fixed point theorem for digital images, Fixed Point Theory Appl. 253 (2013), 1-13.

[10] S. Eilenberg, D. Montgomerry, Fixed point theorems for mulivalued transformations, Amer. J. Math. 58 (1946), $214-222$.

[11] G. Fournier, L. Górniewicz, The Lefschetz fixed point theorem for some noncompact multivalued mappings, Fund. Math. 94 (1977), 245-254.

[12] D.L. Gonçalves, J. Guaschi, Fixed points of $n$-valued maps, the fixed point property and the case of surfraces - a braid approach, Indag. Math. 29 (2018), 91-124.

[13] L. Górniewicz, Topological Fixed Point Theory of Multivalued Mappings, second edition, Springer 2006.

[14] L. Górniewicz, A Lefschetz-type fixed point theorem, Fund. Math. 88 (1975), 103-115.

[15] L. Górniewicz, The Lefschetz coincidence theorem, Lecture Notes in Math. 886 (1981), 116-131. 
[16] L. Górniewicz, Homological methods in fixed point theory of multivalued mappings, Dissertationes Math. 129 (1976), 1-66.

[17] L. Górniewicz, A. Granas, Fixed point theorems for multivalued mappings of ANR-s, J. Math. Pures Appl. 49 (1970), 381-395.

[18] L. Górniewicz, A. Granas, Some general theorems in coincidence theory, J. Math. Pures Appl. 60 (1981), 361-373.

[19] L. Górniewicz, Danuta Rozploch-Nowakowska, The Lefschetz fixed point theory for morphisms in topological vector spaces, Topol. Methods Nonlinear Anal. 20 (2002), 215-233.

[20] A. Granas, J. Dugundji, Fixed Point Theory, Springer 2003.

[21] R. Brown, M. Furi, L. Górniewicz and B. Jiang, Handbook of Topological Fixed Point Theory, eds, Springer 2005, 43-82; $217-264$.

[22] J. Jezierski, An example of finitely valued fixed point free map, Uniwersity Gdansk 6 (1987), 87-92.

[23] D. Miklaszewski, A fixed point theorem for multivalued mappings wit non-acyclic values, Lecture Notes in Nonlinear Analysis 17 (2001), 125-131.

[24] B. O'Neill, A fixed point theorem for multivalued functions, Duke Math. J. 14 (1947), 689-693.

[25] D. Pastor, A remark on generalized compact maps, Stud. Univ. Žilina Math. Ser.13 (2001), 147-155.

[26] H.W. Seigberg, G. Skordev, Fixed point index and chain approximation, Pacific J. Math. 102 (1982), 455-486.

[27] R. Skiba, M. Ślosarski, On a generalization of absolute neighborhood retracts, Topology Appl. 156 (2009), $697-709$.

[28] F. Von Haesler, H.-O. Peitgen, G. Skordev, Lefschetz fixed point theorem for acyclic maps with multiplicity, Topol. Methods Nonlinear Anal. 19 (2002), 339-374. 